\title{
Wie Patienten Wickelanwendungen (ein)schätzen: Ergebnisse einer Umfrage in einem anthroposophischen Akutspital
}

\author{
Ana Paula Simões-Wüst ${ }^{a} \quad$ Georg Saltzwedel $^{\mathrm{a}}$ \\ ${ }^{a}$ Forschungsabteilung, \\ ${ }^{\mathrm{b}}$ Abteilung für Pflegeentwicklung, Paracelsus-Spital Richterswil, Schweiz
}

\section{Schlüsselwörter}

Wickel · Anthroposophische Medizin · Pflege · Umfrage

\section{Zusammenfassung}

Hintergrund: Wickelanwendungen bilden einen festen Bestandteil in der anthroposophischen Medizin und Pflege. Einerseits sollen sie zur Linderung akuter wie chronischer Beschwerden und andererseits zur gezielten Unterstützung von Medikamentenwirkung und Therapien beitragen. Patienten und Methode: Zwischen April 2008 und Februar 2009 wurden an alle stationär behandelten Patienten nach deren Spitalaustritt insgesamt 900 Fragebogen zur Anwendung von Wickeln versendet. Von diesen wurden 394 Fragebögen retourniert (44\%), davon 233 von Patienten, die mindestens einmal während ihres Spitalaufenthalts eine Wickelanwendung bekommen hatten (59\%). 221 Patienten waren volljährig und gaben an, die Wickelwirkung bewerten zu können; die Angaben dieser Gruppe wurden für die vorliegende Auswertung verwendet. Ergebnisse: 70\% der Patienten nahmen eine deutliche oder sehr deutliche Verbesserung ihres Zustands wahr, die sie den Wickeln zuschrieben. In 71\% der Fälle war diese Verbesserung kurzfristig (für den Moment oder für den Tag). Die Mehrheit der Patienten konnte sich durch die Wickel erholen: Für 33\% war die Erholung deutlich, für 36\% gut, für 16\% hervorragend. Der Erfolg der Wickel, der durch vier verschiedene, sich ergänzende und untermauernde Fragen erfasst wurde, wurde von $30 \%$ der Patienten als deutlich, von $30 \%$ als hoch und von $16 \%$ als sehr hoch eingestuft. Bei kritischerem Gesundheitszustand wurde die Wickelwirkung als etwas weniger erfolgreich eingeschätzt. Die Frauenpopulation - am stärksten die Patientinnen, die aufgrund einer Mutterschaft das Spital aufsuchten - schätzte die Wickelwirkung tendenziell positiver ein als die Männerpopulation. Schlussfolgerung: Zusammenfassend zeigt die hier beschriebene Umfrage auf, dass die Mehrheit der Patienten eine hohe Wirksamkeit der Wickel wahrgenommen hat.

\author{
Keywords \\ Packs · Anthroposophic medicine $\cdot$ Nursing $\cdot$ Survey
}

\section{Summary}

How Patients Value Packs: Results of a Survey in an Anthroposophic Hospital Background: Packs constitute an integral part of anthroposophic medicine and care. On the one hand, they are expected to alleviate acute and chronic pain, on the other hand, they are used for supporting the effects of defined medications and therapies. Patients and Method: Between April 2008 and February 2009, a total of 900 questionnaires about the patients' experiences with packs were sent to all hospitalized patients upon their hospital discharge. Of those, 394 questionnaires were returned (44\%), with 233 patients stating that they had been treated with at least one pack during their hospital stay (59\%). 221 patients were of full age and considered themselves to be able to evaluate the effect of the packs; the data corresponding to this group have been considered in the present analysis. Results: $70 \%$ of the patients perceived a considerable or very considerable improvement of their state of health, which they attributed to the packs. In $71 \%$ of the cases, this improvement was short-term (for the moment or for the day). Most of the patients could recover well with the packs: the recovery was considerable for $33 \%$ of them, good for $36 \%$ and outstanding for $16 \%$. The success of the packs, which was inquired by four different questions complementing and corroborating each other, was characterized as being considerable by $30 \%$ of the patients, as high by $30 \%$ and as very high by $16 \%$. Patients in a rather critical health state assessed the effects of the packs as somewhat less successful. The female population - particularly those patients who had consulted the hospital because of maternity - tended to assess the effects of the packs as more positive than the male population. Conclusion: In summary, the present survey shows that the majority of the patients perceived the effectiveness of the packs as high.
Dr. Ana Paula Simões-Wüst

Forschungsabteilung, Paracelsus-Spital Bergstrasse 16, 8805 Richterswil, Schweiz simoes@paracelsus-spital.ch 


\section{Mots-clés}

Cataplasme · Médecine anthroposophique · Soins • Questionnaire

\section{Résumé}

Comment les patients apprécient l'utilisation de cataplasmes: résultats d'un questionnaire dans un hôpital anthroposophique

Contexte: L'utilisation de cataplasmes forme un élément établi dans les soins et médecine anthroposophique. D'un côté, elles doivent permettre de soulager les douleurs chroniques ou aigues; et d'un autre côté, contribuer à ce que les médicaments et traitement ciblés fassent effet. Patients et méthode: Entre avril 2008 et février 2009, 900 questionnaires sur l'utilisation des cataplasmes ont été envoyés à tous les patients traités sur place après leur sortie d'hôpital. 394 questionnaires ont été retournés (44\%), comprenant 233 patients qui avaient reçu au moins une fois durant leur séjour hospitalier un traitement avec cataplasmes (59\%). 221 patients étaient majeurs et ont pu juger l'utilisation de cataplasmes. Les déclarations de ce groupe ont été utilisées pour l'estimation. Résultats: $70 \%$ des patients ont constaté une amélioration nette ou très nette de leur état et l'attribuent aux cataplasmes. Dans $71 \%$ des cas, cette amélioration était à court terme (sur le moment ou pour la journée). La majorité des patients ont pu se rétablir grâce aux cataplasmes: pour $33 \%$, le rétablissement était net, satisfaisant pour $36 \%$ et excellent pour $16 \%$. L'efficacité des cataplasmes, déterminée grâce à quatre questions différentes à compléter et à étayer, a été estimée comme nette par $30 \%$ des patients, haute par $30 \%$ et très haute par $16 \%$. Pour les états de santé critiques, I'utilisation de cataplasmes a été jugée moins efficace. Les femmes, dont la majorité était au service maternité, ont eu tendance à évaluer l'effet des cataplasmes de manière plus positive que les hommes. Conclusions: En conclusion, le questionnaire décrit ici montre que la plupart des patients ont reconnu l'efficacité des cataplasmes.

\section{Einleitung}

Wickel als eine Form äusserlicher Anwendung, bei der ein Körperteil mit der heilsamen Substanz bedeckt und mit einem Tuch umhüllt wird, kommen innerhalb der anthroposophischen Medizin häufig zum Einsatz. Sie sollen entspannend oder schmerzstillend wirken, die Selbstheilungskräfte anregen und die Reaktion auf Medikamente sowie auf zusätzliche Therapien fördern. Das Spektrum der benutzten umhüllenden Materialien wie auch der aufgetragenen Substanzen ist vielfältig [1]. Sie reichen von mineralischen Substanzen wie Quarz und Schwefel oder metallischen Substanzen wie Kupfer und Gold über pflanzliche Extrakte wie z.B. Kamille, Arnika, Schafgarbe und Senf bis hin zu Produkten tierischer Herkunft wie Quark und Honig. Wickel können bei nahezu allen akuten und chronischen Erkrankungen sowohl in der häuslichen [2,3] als auch in der professionellen Krankenpflege eingesetzt werden [1]. Die dazugehörige Fach- und Laienliteratur ist zahlreich (grossteils unter www.wickel.biz aufgelistet). Dagegen bleibt die Wirksamkeit der verschiedenen Anwendungen wenig bewiesen: In einer vor kurzem veröffentlichten systematischen Literatursuche [4] zu rein pflegerischen, interventionellen Publikationen in Deutsch und Englisch über Wickel und Auflagen wurden zwar 14 Studien identifiziert, jedoch wurde ihre Aussagekraft wegen methodischer Schwächen als begrenzt eingeschätzt. Die Ergebnisse dieser Studien deuten sowohl insgesamt auf positive Effekte der Wickel bei Schmerzreduzierung und Erhöhung des Wohlbefindens als auch im Einzelnen auf Verbesserungen bei einigen physiologischen Parametern hin [4].

Wie im Rahmen eines Netzwerks Onkologie erfasst, werden Wickel sehr häufig als Zusatztherapien bei Krebspatienten benutzt [5]. Dabei werden in den symptombezogenen pflegerischen Anwendungen die verschiedenen Wickel ebenso wie andere Arten der äusseren Anwendungen je nach Krankheitsphase und Zustand differenziert eingesetzt [6]. Bei Krebs und seinen Begleitsymptomen wie Angst, Unruhe, Übelkeit oder Obstipation ist vorwiegend ein wärmender Effekt erwünscht, weswegen warme oder heisse Wickel bevorzugt werden, während kühle und kalte Wickel für Ödeme oder akute Entzündungen als geeignet erscheinen [6]. Auch in der Schmerztherapie spielen Wickel eine wichtige Rolle. Bei stumpfen Verletzungen und bei Hämatomen werden Arnika-Wickel empfohlen, bei Arthritis Arnika-Heilerde-Wickel; wenn der Schmerz mit entzündlichen Prozessen assoziiert ist, können Quarkwickel hilfreich sein [7]. Darüber hinaus werden Wickel auch eingesetzt, um bestimmte Organe in ihrer Tätigkeit anzuregen [8], sowie in der Pflege von Menschen mit Demenz (www.wickel.biz/literaturartikel.htm) und um Fieber zu senken (www.kinderklinik.at/1389.php). $\mathrm{Zu}$ letzterem Aspekt zeigte eine Umfrage, dass die Mehrheit (70\%) der Eltern eines fiebrigen Kindes eine Verbesserung von dessen Zustand und Wohlbefinden durch Wickel wahrgenommen hat (www.kinderklinik.at/1389.php).

Das Paracelsus-Spital Richterswil ist ein Akutspital mit den Fachgebieten Geburtshilfe und Gynäkologie, Innere Medizin, Integrative Onkologie und Hämatologie, Chirurgie und Urologie und wurde kürzlich als Kompetenzzentrum für Palliativmedizin anerkannt. Dieses unweit von Zürich gelegene Krankenhaus mit 48 Betten bietet ergänzend zur konventionellen Medizin komplementärmedizinische Methoden mit dem Schwerpunkt anthroposophische Medizin an. Im Rahmen des Qualitätsmanagements wird routinemässig an jeden stationär behandelten Patienten nach Spitalaustritt ein Patientenfragebogen verschickt. Damit holt sich das Spital eine Rückmeldung der Patienten über ihre Zufriedenheit mit den verschiedenen vom Spital angebotenen Dienstleistungen. Zwischen April 2008 und Februar 2009 wurde unter 
anderem ein Fragebogen über die wahrgenommene Wirksamkeit von Wickelanwendungen an die Patienten versendet. In der vorliegenden Studie wurden die Antworten der Patienten auf diesen Fragebogen ausgewertet. Dies liefert Hinweise zur Wirksamkeit von Wickelanwendungen aus Patientensicht.

\section{Methode}

Der Fragebogen wurde zwischen April 2008 und Februar 2009 sämtlichen im Paracelsus-Spital Richterswil stationär behandelten Patienten nach deren Austritt zugestellt $(n=900)$. Der Fragebogen beinhaltete folgende Fragen:

1) «Wie würden Sie Ihren (aktuellen) Gesundheitszustand im Allgemeinen beschreiben?» (mögliche Antworten: «ausgezeichnet», «sehr gut», «gut», «weniger gut», «schlecht»);

2) «Haben die Wickel Ihr Wohlbefinden gefördert?» («nein», «für den Moment», «für den Tag», «für 2-3 Tage», «für 1 Woche», «für länger als 1 Woche», «weiss nicht»);

3) «Wie stark konnten Sie sich durch die Wickel erholen?» («gar nicht», «wenig», «mässig», «deutlich», «gut», «hervorragend»);

4) «Welchen Beitrag zum Gesamtbehandlungsziel haben die Wickel geleistet?» («keinen», «minimal», «mässig», «deutlich spürbar», «wichtig», «sehr wichtig», «weiss nicht»);

5) «Welche heilenden oder vorbeugenden Veränderungen haben sich durch die Wickel ergeben?» («Zustand deutlich verschlechtert», «Zustand leicht verschlechtert», "Zustand unverändert», «leichte Verbesserung», «deutliche Verbesserung», «sehr deutliche Verbesserung»);

6) «Bewerten Sie die Anwendung als Erfolg?» («nein», «ein wenig», «mässig», «deutlich», «stark», «voll und ganz», «weiss nicht»);

7) «Wurde das durch die Wickel angestrebte Behandlungsziel erreicht?» («nein, gar nicht», «ein wenig», «mittelmässig», «deutlich», «gut», «sehr gut», «weiss nicht»);

8) «Welchen Anteil haben die Wickel am Behandlungserfolg?» («gar keinen», «ein wenig», «mittelmässig», «deutlich», «grossen Anteil», «sehr grossen Anteil»);

9) «Welche Veränderungen haben sich durch die anthroposophischen Medikamente ergeben?» («Zustand deutlich verschlechtert», «Zustand leicht verschlechtert», "Zustand unverändert», «leichte Verbesserung» «deutliche Verbesserung», «sehr deutliche Verbesserung», «weiss nicht»);

10) «Wie wertschätzend im Kontakt, mit innerer Beteiligung am praktischen Tun und um Ihr Wohlbefinden bemüht ist Ihnen das Pflegepersonal begegnet?» («nein, gar nicht», «ein wenig», «mittelmässig», «deutlich», «gut», «sehr gut»);

11) «Wie bewerten Sie die Kompetenz des Pflegepersonals, das Ihnen die Wickel verabreicht hat?» («ungenügend», «mangelhaft», «ausreichend», "zufriedenstellend», «gut», «sehr gut»);

12) «Wie gross schätzen Sie Ihr Wissen über die Wirksamkeit der Wickel ein?» («weiss gar nichts», «gering», «mässig», «ziemlich», «sehr»);

13) Wie viele Wickelanwendungen haben Sie während Ihres Spitalaufenthalts erhalten?

Mit den fehlenden Werten wurde wie folgt umgegangen: Wenn bei einem Patient maximal 2 Fragen unbeantwortet blieben (nicht ausgefüllt oder mit «weiss nicht» markiert), so wurden diese mit dem Durchschnittswert der verbleibenden Fragen derselben Person ergänzt. Für alle übrigen Fälle wurden die fehlenden Werte aus dem Durchschnittswert aller Befragten bestimmt. 25 Werte wurden auf diese beiden Arten ersetzt. Handschriftliche Bemerkungen blieben unberücksichtigt, ausser bei der Frage nach der Anzahl der erhaltenen Wickel. Hier wurde wie folgt quantifiziert «mehrere Anwendungen» wurden als 10 Anwendungen gewertet (2 Fälle), «viele Anwendungen» wurden als 20 Anwendungen klassifiziert (2 Fälle).

\section{Ergebnisse}

\section{Patienten}

Von den 900 versandten Fragebogen wurden 394 zurückgeschickt (44\%). Gemäss den 394 retournierten Fragebögen gaben 233 Patienten an, eine Wickelanwendung erhalten zu haben (59\%); 161 erhielten keine solche Anwendung (41\%). 11 der Patienten, die Wickel verabreicht bekamen, fühlten sich nicht in der Lage, den Wirkungserfolg zu beurteilen (5\%), und wurden von der Auswertung ausgeschlossen. Auch der Fragebogen einer 7-jährigen Patientin wurde von der Auswertung ausgeschlossen. Entsprechend konnten 221 Fragebögen - davon 196 von weiblichen und 25 von männlichen Patienten, die Wickelanwendungen bekommen hatten und sich in der Lage fühlten, sie zu bewerten - ausgewertet werden $(n=221$; entspricht $56 \%$ der zurückgesandten Fragebögen); die Patienten waren durchschnittlich 49,6 \pm 19,2 Jahre alt (Mittelwert \pm Standardabweichung/SD; Spannweite 18-92). Alle in dieser Studie ausgewerteten Angaben entsprechen den Meinungen der Patienten und wurden im Rahmen dieser Studie keiner zusätzlichen unabhängigen ärztlichen Beurteilung unterzogen.

Ein grosser Teil der Patienten $(83 ; 37,6 \%)$ suchte das Spital aufgrund einer Geburt auf, ein weiterer recht grosser Teil $(63 ; 28,5 \%)$ aufgrund innerer Krankheiten. Erwähnt wurden auch chirurgische Eingriffe (30, 13,6\%), gynäkologische Erkrankungen $(25 ; 11,8 \%)$ und gynäko-chirurgische Eingriffe (20; 8,6\%).

Die Einstiegsfrage des Fragebogens lautete: «Wie würden Sie Ihren (aktuellen) Gesundheitszustand im Allgemeinen beschreiben?» Mit dieser Frage wurde der subjektiv von den Patienten zum Zeitpunkt des Ausfüllens des Fragebogens wahrgenommene Gesundheitszustand erfasst. Die meisten Patienten $(104 ; 47,1 \%)$ antworteten mit «gut», 46 (20,8\%) mit «sehr gut» und $17(7,7 \%)$ mit «ausgezeichnet». Dagegen schätzten 41 (18,6\%) Patienten ihren Gesundheitszustand als «weniger gut» ein und 13 (5,9\%) als «schlecht». Dann wurde nach der Anzahl der Wickel gefragt, die den Patienten während ihres Spitalaufenthalts von Pflegenden angelegt worden waren. Die grösste Gruppe der Patienten $(102 ; 46,2 \%)$ gab an, 1-5 Wickel erhalten zu haben. 67 (30,3\%) Patienten hatten 6-10 Wickel bekommen, 26 (11,8\%) Patienten 11-15, 13 (5,9\%) Patienten 16-20 und 13 (5,9\%) Patienten 21 oder mehr Wickel. Der Median lag damit bei $6 \pm 0,5$ (Standardabweichung des Mittelwerts, SEM) Wickeln.

Bei der Frage nach dem eigenen Wissen zur Wirksamkeit von Wickeln gaben 8 (3,6\%) Patienten an, nichts über die Wirksamkeit zu wissen, während 213 Patienten angaben, etwas darüber zu wissen. Die grösste Gruppe der Patienten $(96 ; 43,4 \%)$ stufte ihr Wissen als «ziemlich hoch» ein, 42 (19,0\%) sogar als «sehr hoch». 
Wahrnehmung des Pflegenden von Wickelanwendungen durch die Patienten

Wickelanwendungen bedeuten immer auch persönlichen Kontakt zwischen Patient und Pflegefachperson. Denkbar ist deshalb, dass die Haltung der Pflegenden die Anwendungswirkung beeinflusst. Eine Frage bezog sich direkt auf die Umsetzung des Pflegeleitbilds des Paracelsus-Spitals Richterswil, auch wenn der entsprechende Text eigentlich drei Aspekte beinhaltet. Im Pflegeleitbild heisst es, die Pflegenden mögen wertschätzend im Kontakt, mit innerer Beteiligung am praktischen Tun und um des Patienten Wohlbefinden bemüht agieren. Die Frage hierzu lautete: «Wie wertschätzend im Kontakt, mit innerer Beteiligung am praktischen Tun und um Ihr Wohlbefinden bemüht ist Ihnen das Pflegepersonal begegnet?» Für 152 (68,8\%) Patienten entsprachen die Pflegenden dem Leitbild «sehr gut», für $54(24,4 \%)$ «gut». Nur $3(1,4 \%)$ Patienten gaben «nein, gar nicht» bis «ein wenig» an, $4(1,8 \%)$ «mittelmässig» und $8(3,6 \%)$ «deutlich». Die Patienten wurden gebeten, die Kompetenz der Pflegefachpersonen, die ihnen die Wickel verabreicht hatten, zu bewerten. Dabei wurde die Bewertung «sehr gut» 119-mal abgegeben (53,8\% der Patienten). 74 (33,5\%) Patienten kreuzten «gut» an, $26(11,8 \%)$ «ausreichend» oder «zufriedenstellend» und $2(0,9 \%)$ «ungenügend» oder «mangelhaft».

\section{Wirkung der Wickel}

Bei allen im Folgenden abgebildeten Ergebnissen geht es um die subjektive Einschätzung der Patienten zu Wickelanwendungen, präziser ausgedrückt um deren gespürte Wirkung. Lediglich der Schwerpunkt der einzelnen Fragen wurde unterschiedlich gewählt. In Abbildung 1 wird der Zeitraum dargestellt, über den die Wickelanwendungen im Anschluss an die Applikation als angenehm empfunden wurden. Die meisten Patienten erfuhren eine kurzfristige Förderung ihres Wohlbefindens (157 Patienten (71,0\%) bis zu einem Tag lang); bei einem Teil der Patienten war dieser Effekt über mehrere Tage oder sogar für länger als eine Woche spürbar. Abbildung 2 beschreibt heilende oder vorbeugende Veränderungen, welche die Patienten nach der Wickelanwendung gespürt haben. Die Mehrheit der Patienten spürte eine Verbesserung, davon 98 (44,3\%) eine «deutliche Verbesserung» und 35 (15,8\%) eine "sehr deutliche Verbesserung». Nach den Angaben der Patienten hatten sie sich mehrheitlich durch die Wickel «deutlich», «gut» oder «hervorragend» erholt (Abb. 3).

In Abbildung 4 sind die gemittelten Werte der Skala «Erfolg der Wickelanwendungen» (221 Antworten bei allen Fragen) angegeben. Berechnet wurde diese Skala aus den Antworten auf die vier Einzelfragen «Welchen Beitrag zum Gesamtbehandlungsziel haben die Wickel geleistet?»,
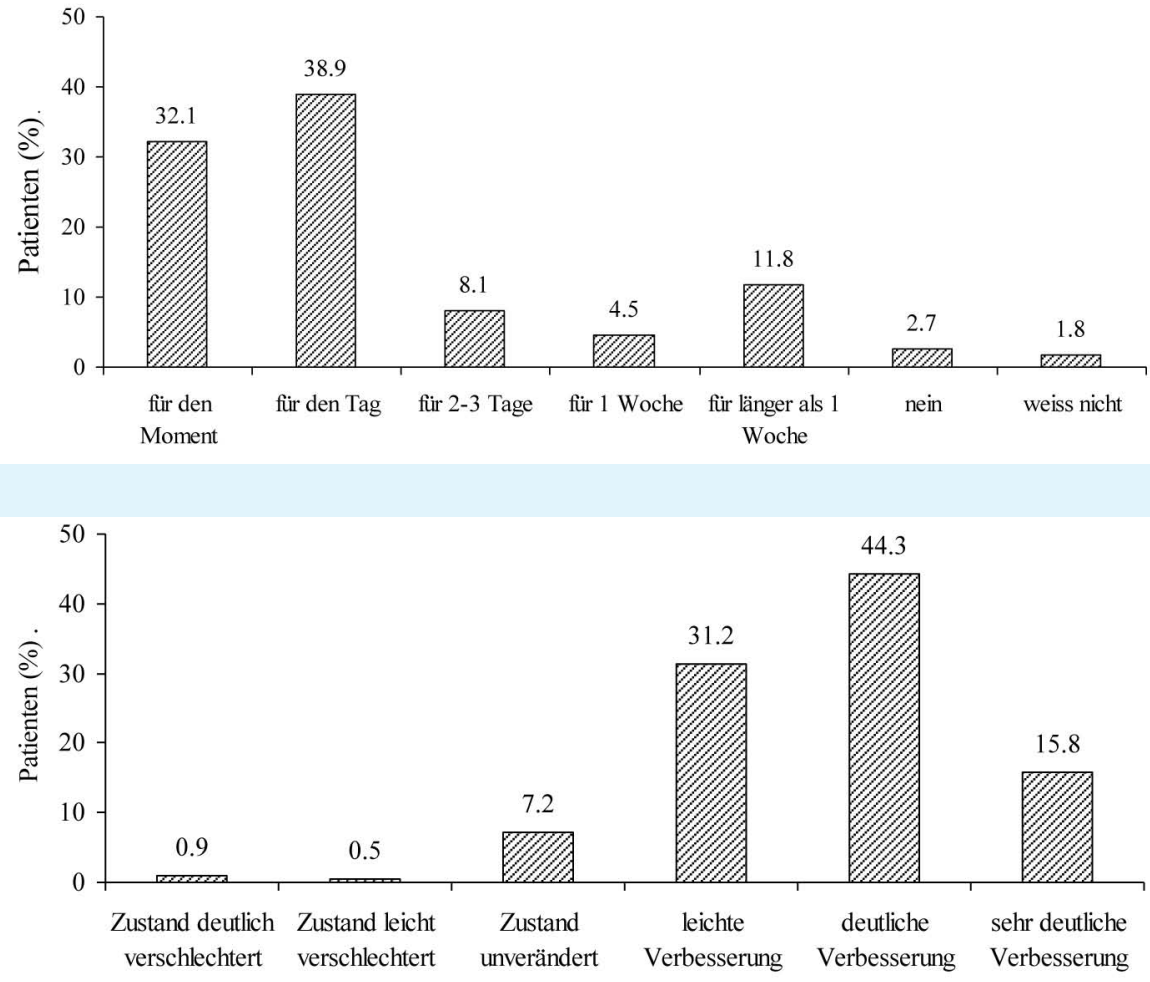

Abb. 1. Antwortverteilung auf die Frage: «Haben die Wickel Ihr Wohlbefinden gefördert?»
Abb. 2. Antwortverteilung auf die Frage: «Welche heilenden oder vorbeugenden Veränderungen haben sich durch die Wickel ergeben?» 
Abb. 3. Antwortverteilung auf die Frage: «Wie stark konnten Sie sich durch die Wickel erholen?»

Abb. 4. Antwortverteilung auf die ermittelte Skala «Erfolg der Wickelanwendungen».
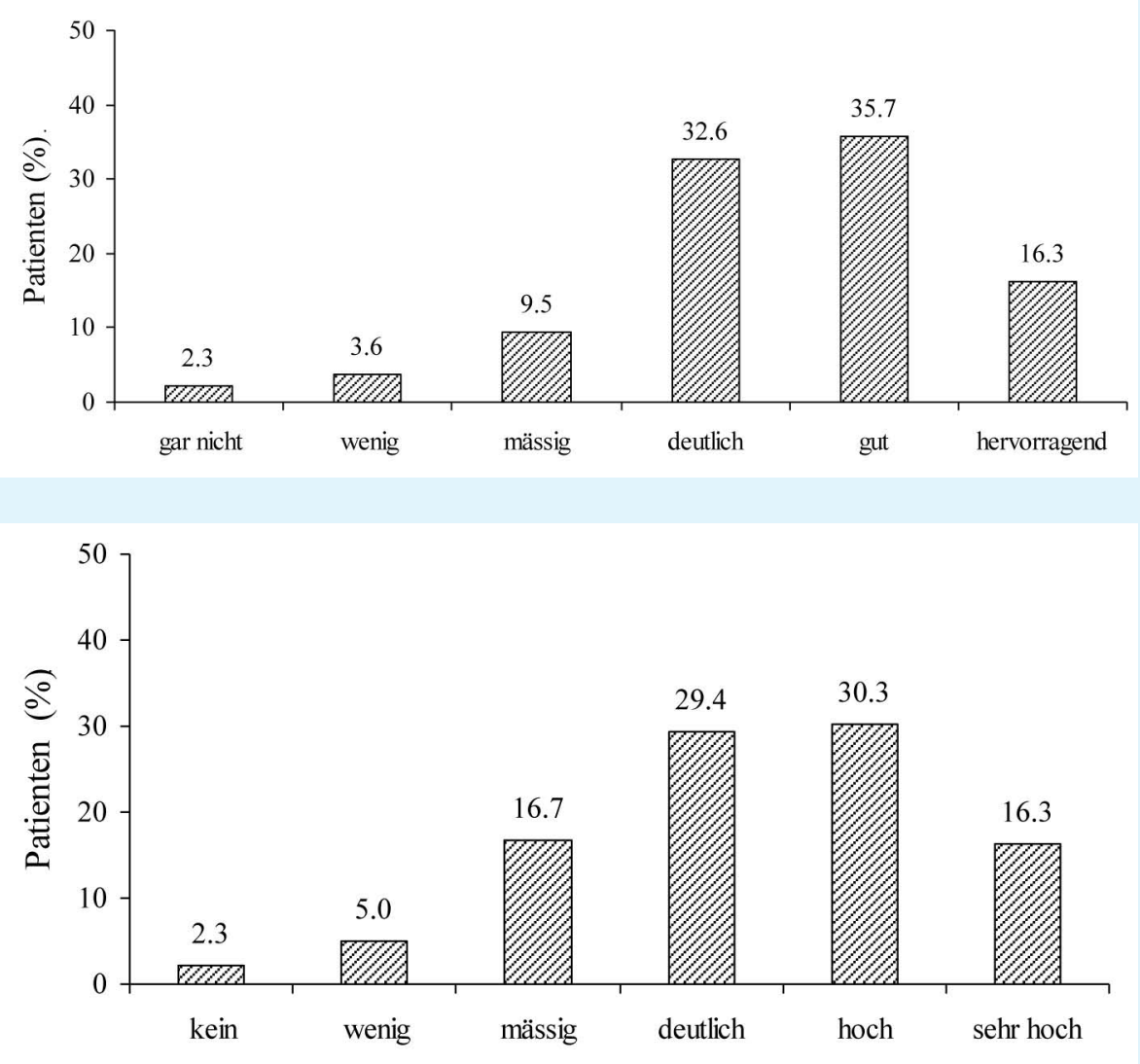

«Bewerten Sie die Anwendung als Erfolg?», «Wurde das durch die Wickel angestrebte Behandlungsziel erreicht?» und «Welchen Anteil haben die Wickel am Behandlungserfolg?», mit denen der Erfolg der Wickelanwendungen gemessen werden sollte. Alle vier Fragen boten die gleiche Anzahl von Antwortmöglichkeiten. Die Ergebnisse der daraus resultierenden Skala zeigen, dass die Mehrheit der Patienten (168 von 222; 76,0\%) einen deutlichen, hohen oder sehr hohen Erfolg der Wickel spürte. Nur 5 (2,3\%) Patienten nahmen keinen Erfolg wahr.

\section{Subgruppen}

Wickelwirkungen bei Patientinnen, die das Spital aufgrund einer Mutterschaft aufgesucht hatten

Diese Subgruppe wurde gewählt, da sie die meisten Patienten umfasste. Ausnahmslos alle Patientinnen dieser Gruppe $(n=83)$ gaben an, sich durch die Wickel erholt $\mathrm{zu}$ haben - 9 wenig bis mässig, 74 deutlich bis hervorragend. Bei der gemittelten Skala «Erfolg der Wickelanwendungen» bewerteten 16 Patientinnen der Subgruppe die Anwendung als «sehr gut», 28 als «gut», 27 als «deutlich» und 12 als «minimal» bis «mässig».

\section{Geschlechtsunterschiede}

Von den 221 Patienten, deren Antworten in diesem Artikel dargestellt sind, waren 196 Frauen und 25 Männer. Obwohl sich die beiden Patientengruppen in ihrer Anzahl deutlich unterscheiden, zeigte sich, dass die Frauenpopulation die Wickelwirkung deutlich positiver einschätzte als die Männerpopulation. Während 31,6\% der Frauen den Erfolg der Anwendung als "gut» und 16,8\% diesen als «sehr gut» bezeichneten, waren es bei den Männern 20,0\% bzw. 12,0\%. $40,0 \%$ der männlichen Patienten gaben an, die Anwendung sei «gar nicht» bis «mässig» erfolgreich gewesen; bei den Frauen waren dies lediglich $21,9 \%$.

\section{Wirksamkeitsunterschiede in Abhängigkeit vom Gesundheitszustand}

Um der Frage nachzugehen, ob die Wickelanwendungen immer gleich wirken, unabhängig vom aktuellen Gesundheitszustand des Patienten, wurden drei Patientengruppen nachträglich gebildet. Die Patienten der ersten Gruppe hatten auf dem Fragebogen angegeben, es gehe ihnen zum Zeitpunkt des Ausfüllens des Fragebogens «ausgezeichnet» oder «sehr gut» $(n=63)$. Die zweite Patientengruppe hatte auf die Frage nach dem Gesundheitszustand «gut» geantwortet $(\mathrm{n}=104)$. Den Patienten der dritten Gruppe ging es nach Selbstauskunft eher «weniger gut» oder «schlecht» $(n=54)$. 


\begin{tabular}{lll}
\hline Gesundheitszustand & Antwortverteilung, \% & \\
\cline { 2 - 3 } & $\begin{array}{l}\text { gar nicht } \\
\text { bis mässig }\end{array}$ & $\begin{array}{l}\text { deutlich bis } \\
\text { hervorragend }\end{array}$ \\
\hline Ausgezeichnet oder sehr gut & 9,5 & 90,5 \\
Gut & 11,5 & 88,5 \\
Weniger gut oder schlecht & 18,5 & 81,5 \\
\hline
\end{tabular}

\begin{tabular}{llll}
\hline Gesundheitszustand & \multicolumn{2}{l}{ Antwortverteilung, \% } & \\
\cline { 2 - 4 } & verschlechtert & $\begin{array}{l}\text { unverändert } \\
\text { bis leicht besser }\end{array}$ & $\begin{array}{l}\text { deutlich bis sehr } \\
\text { deutlich besser }\end{array}$ \\
\hline Ausgezeichnet oder sehr gut & 1,6 & 27,0 & 71,4 \\
Gut & 1,9 & 36,6 & 61,5 \\
Weniger gut oder schlecht & 0,0 & 57,4 & 42,6 \\
\hline
\end{tabular}

\begin{tabular}{lccccc}
\hline Gesundheitszustand & \multicolumn{2}{l}{ Antwortverteilung, \% } & & \\
\cline { 2 - 6 } & kein & mässig & deutlich & hoch & sehr hoch \\
\hline Ausgezeichnet oder sehr gut & 1,6 & 9,5 & 28,6 & 33,3 & 27,0 \\
Gut & 7,7 & 14,4 & 28,8 & 38,5 & 10,6 \\
Weniger gut oder schlecht & 13,0 & 31,4 & 27,8 & 14,8 & 13,0 \\
\hline
\end{tabular}

Tab. 1. Wirksamkeitsunterschiede in Abhängigkeit vom Gesundheitszustand: «Wie stark konnten Sie sich durch die Wickel erholen?»

Tab. 2. Wirksamkeitsunterschiede in Abhängigkeit vom Gesundheitszustand: «Welche heilenden oder vorbeugenden Veränderungen haben sich durch die Wickel ergeben?»

Tab. 3. Wirksamkeitsunterschiede in Abhängigkeit vom Gesundheitszustand: Ergebnisse der ermittelten Skala «Erfolg der Wickelanwendungen»
Die Daten dieser drei Gruppen werden in den Tabellen 1-3 bezüglich zwei Fragen und der gemittelten Werte der Skala «Erfolg der Wickelanwendungen» dargestellt. $\mathrm{Da}$ es sich um einen direkten Vergleich handelt, werden die Daten aus Übersichtsgründen nur als relative Werte (\%) gezeigt. Tendenziell zeigt sich, dass Patienten Antworten wie «hervorragend» oder «sehr hoch» desto seltener ankreuzten, je schlechter ihr Gesundheitszustand war. Bei der Frage «Welche heilenden oder vorbeugenden Veränderungen haben sich durch die Wickel ergeben?» sowie bei der ermittelten Skala «Erfolg der Wickelanwendungen» waren die Unterschiede zwischen den verschiedenen Gruppen ersichtlich. Dennoch wurde eine deutliche bis hervorragende Erholung in allen Gruppen sehr häufig erzielt.

\section{Diskussion}

Als die Patienten die Fragebogen ausfüllten, schätzten sie ihren eigenen Gesundheitszustand sehr unterschiedlich ein. Die Einweisungsgründe deuten weiter darauf hin, dass Gesundheits- und Lebensumstände der an dieser Umfrage teilnehmenden Patienten sehr vielfältig waren. Diese Vielfalt war aufgrund des medizinischen Angebots des Paracelsus-Spitals Richterswil zu erwarten. Auffallend hoch war die Anzahl der Patientinnen, die das ParacelsusSpital aufgrund einer Mutterschaft aufgesucht haben. Wie die Subgruppen-Analyse darstellt, schätzten vor allem diese Patientinnen die Wirkung von (typischerweise Gold-, Lavendel-, seltener Kümmelöl-)Wickeln. Bei dieser sehr positiven Einschätzung spielt möglicherweise der vorwiegend erfreuliche Charakter eines Spitalaufenthalts wegen einer Geburt und auch die Tatsache eine Rolle, dass diese Population meistens nicht an schwerwiegenden Erkrankungen leidet, sondern eine rasche, exponentielle Verbesserung der Beschwerden erfährt. Aber auch die Frauenpopulation insgesamt schätzte die Wickelwirkung deutlich positiver ein als die Männerpopulation, was auf eine geschlechtsspezifische Einstellung hindeutet. Mit schlechterem Gesundheitszustand der Patienten wurde die Wickelwirkung als etwas weniger erfolgreich eingeschätzt, obwohl in vielen Fällen ein Erholungseffekt trotzdem angegeben wurde. Weiter scheint das Bemühen der in der anthroposophischen Medizin tätigen Pflegefachpersonen um einen adäquaten, einfühlsamen Umgang mit Patienten (entsprechendes Zitat aus dem Pflegeleitbild: «wertschätzend im Kontakt, mit einer inneren Beteiligung am praktischen Tun und um das Wohlbefinden der Patienten bemüht») sehr gut umgesetzt worden zu sein. Die Mehrzahl $(68,9 \%)$ der Patienten fand, dass die Pflegenden sehr gut dem Leitbildzitat entsprachen.

Eingeschränkt wird die Aussagekraft der vorliegenden Studie durch die zum Teil schwer voneinander zu differenzierenden vorgegebenen Antwortmöglichkeiten wie auch deren etwas asymmetrische Verteilung. Darüber hinaus können die erwähnten Verbesserungen nicht mit Sicherheit auf die Wickelanwendungen zurückgeführt werden, da die Patienten gleichzeitig zu den Wickeln mit anderen Therapien/Heilmitteln behandelt wurden und Aufmerksamkeit durch die Pflegenden erfuhren. Auch können wir eine wohlwollende, grundlegend positive Einstellung der Patienten gegenüber der anthroposophischen Medizin nicht ausschliessen: Es ist bekannt, dass das Paracelsus-Spital Richterswil diese Form der Komplementärmedizin anbietet, und dies dürfte für viele Patienten bei der Spitalsuche eine Rolle gespielt haben. Positiv auf die Ergebnisse der Studie wirken sich die genügend hohe 
Zahl der Befragten und das Ausschliessen von Patienten, die sich nicht in der Lage fühlten, den Wirkungserfolg zu beurteilen, aus. Das von den Patienten angegebene Wissen über Wickelanwendungen lässt entweder auf ein mitgebrachtes Vorwissen oder auf Aufklärung durch die Pflegefachpersonen schliessen; allerdings muss auch mögliche Selbstüberschätzung (soziale Erwünschtheit) bedacht werden. Angegebenes Wissen zusammengenommen mit der Tatsache, dass die Patienten durchschnittlich 6 Wickel bekommen hatten, lassen jedoch annehmen, dass die meisten Patienten über genügend Erfahrungen mit dieser Pflegeanwendung verfügten.

Die meisten Patienten verspürten eine heilende oder vorbeugende Veränderung durch die Wickel, die in einer beträchtlichen Fallzahl eine nachhaltige Wirkung hatten. Fast die Hälfte aller Patienten berichtete über einen hohen bis sehr hohen Erfolg. Die Zahl der Patienten, welche die Anwendungen als Erfolg bewerteten, war aber grösser als die derjenigen, welche angaben, eine heilende oder vorbeugende Veränderung durch die Wickel gespürt zu haben (217 vs. 202). Dies deutet darauf hin, dass bei manchen Patienten nicht eine Zustandsverbesserung, sondern eine Zustandserhaltung als positiv wahrgenommen worden ist.

Zusammenfassend zeigt die hier ausgewertete Umfrage auf, dass von der genannten Population der Patienten, die sich zwischen April 2008 und Februar 2009 einer statio- nären Behandlung im Paracelsus-Spital Richterswil unterzogen hatten, die Wirkung von Wickeln mehrheitlich als ziemlich hoch eingeschätzt wurde. Die Anwendung von Wickeln, die charakteristisch für die anthroposophische Medizin ist, wurde bereits von vielen konventionellen Pflege- und Behandlungsinstitutionen aufgegriffen. Die Pflegemethode hielt Einzug in das Ausbildungs- und Weiterbildungswesen für professionelle Pflege $[9,10]$ - unabhängig von einem bestimmten Menschenbild wie z.B. dem der Anthroposophie. Die vorliegende Studie unterstreicht das Anliegen, diese Pflegemassnahme in der pflegerischen Berufsbildung weiter zu fördern und ihre Wirksamkeit sowohl mit qualitativen als auch mit quantitativen Forschungsmethoden zu untersuchen.

\section{Dank}

Für finanzielle Unterstützung danken wir der Firma Weleda AG (Arlesheim, Schweiz) und der Johannes Kreyenbühl Akademie (Dornach, Schweiz).

\section{Disclosure Statement}

Es bestehen keinerlei Interessenkonflikte.

\section{Literatur}

1 Fingado M: Therapeutische Wickel und Kompressen - Handbuch aus der Ita Wegman Klinik, ed 4. Dornach, Natura, 2008.

2 Goebel W, Glöckler M: Kindersprechstunde. Ein medizinisch-pädagogischer Ratgeber. Stuttgart, Urachhaus, 1995.

3 Uhlemayr U: Wickel und Co. - Bärenstarke Hausmittel für Kinder. Burberg, Urs, 2001.

4 Bächle-Helde B: Komplementäre Pflegemethoden und Evidenzbasierung - Eine Literaturstudie am Beispiel von Wickel und Auflagen. Saarbrücken, Dr. Müller, 2010
5 Schad F, Teodoridis C, Albrecht U, Hoffmann G, Jeschke E, Stumpf C, Fricke L, Breitkreuz T, Baute R, Paxino C, Hesse M, Gutsch J, Matthes B, Debus M, Seibt H, Arndt L, Matthes H: Das Netzwerk Onkologie (NO) - Aufbau und Etablierung eines Forschungsverbundes in der anthroposophisch orientierten, onkologischen Medizin. Der Merkurstab 2007;60:234-237.

6 von Dach C, Heine R, Heiligtag H-R: Anthroposophische Pflege von Krebskranken. Der Merkurstab 2009;62:330-343.
7 Girke M: Schmerzverständnis und Schmerztherapie in der Anthroposophischen Medizin. Der Merkurstab 2008;61:419-434.

8 Matthes H: Aspekte zur Therapie der Hepatitis C. Der Merkurstab 1999;52:25-41.

9 Schewior-Popp S, Sitzmann F, Ullrich L: Das Lehrbuch für Pflegende in der Ausbildung, ed 11. Stuttgart, Thieme, 2009

10 Sonn A: Wickel und Auflagen - Alternative Pflegemethoden erfolgreich anwenden, ed 3. Stuttgart, Thieme, 2010. 